\title{
Modelling and experimental validation of the effect of the elastic properties of fabrics on the durability of screen printed e-textiles
}

\author{
A O Komolafe, R N Torah, M J Tudor and S P Beeby \\ Electronics and Computer Science (ECS), University of Southampton, SO17 1BJ, UK \\ E-mail: a.o.komolafe@ soton.ac.uk
}

\begin{abstract}
Fabrics are stiff in tension but highly compliant in compression in the plane of the textile. The effect of these differing elastic properties on the durability of electronics integrated in or on the fabric is still largely unknown because fabric properties are not easily characterized. Using a mathematical model combining classical bea m theory (CBT) and Pierce's fabric cantilever test, this paper models the bending behaviour of a woven fabric and locates its neutral axis (NA) as a basis for developing more durable printed etextiles. The CBT model showed that the difference in the tensile and compressive moduli of a fabric reduces the bending resistance of the fabric and also moves its NA position away from the central axis on the fabric. Results obtained from a Pierce's bending test of four different textile blends of polyester, cotton and lycra indicate textiles can have anisotropic elastic moduli with different values in their warp and weft directions. This results in different NA positions that vary depending upon the direction of the bending forces. Empirical verification of these NAs was achieved by comparing the change in resistance of a set of screen printed piezoresistive strain gauges positioned on and away from the NA during positive and negative bending with a radius of $5 \mathrm{~mm}$. The gauge positioned at a 1 $\%$ distance from the NA position showed approximately $0.3 \%$ change in its electrical resistance in contrast to a $37 \%$ change in its resistance when it was located at a $65 \%$ distance away from the NA.
\end{abstract}

\section{Introduction}

Screen printed e-textiles are textiles that perform intelligent functions such as sensing and actuation depending on the type of functional material printed. E-textiles find applications in medicine, military, fashion and entertainment [1]. Screen printing is particularly attractive for e-textile fabrication because of its simple and versatile fabrication process, offering a high degree of design freedom and already used throughout the textiles industry. Screen printed e-textile prototype examples include light emitting textiles [2, 3], a planar circuit for fashion applications [4] and bio-potential monitoring systems [5, 6]. However the durability of these e-textiles is limited by mechanical effects that degrade the electrical performance of the electronic circuits when they are exposed to the typical stresses arising from, for example, washing and bending.

In the literature, the durability of the screen printed e-textiles was initially enhanced by improving mechanical properties such as the flexibility and elasticity of the electrical interconnections so they can withstand bending stresses [7-9]. Waterproof and abrasion-resistant thermoplastic polymer layers were 
also incorporated into the e-textiles to reduce failures by encapsulating the electrical interconnections and electronic components on the textile [10-14]. Other improvements focused on localizing the functional film on the neutral axis (NA) of the screen printed e-textile where the strain and stress experienced by the functional layer are minimised and ideally zero [12-14]. In [12, 13], the NAs of two different screen printed e-textiles were empirically located by comparing the electrical resistance changes of printed piezoresistive strain gauges. The strain gauges were located at different heights in the printed assembly by varying the screen printed layer thicknesses as shown in figure 1 . The NA position was identified by the strain gauge location that exhibited the smallest change in electrical resistance during bending around a radius of $5 \mathrm{~mm}$. Similarly, Paul et al. [14] attempted to empirically locate a silver conductor on the NA of several screen printed e-textiles but this analysis did not consider the unusual elastic properties of the woven fabric substrates which led to poor correlation between theoretical and empirical results. The resistance changes of the silver conductors after bending indicated that the conductors were strained by the bending and were not located on the NA.

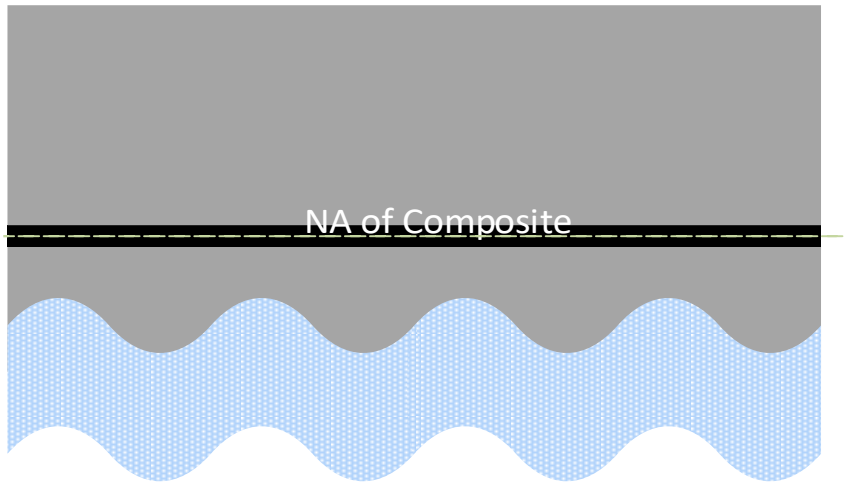

TPU Encapsulation Layer

Gauge Layer

TPU Interface Layer

Woven Fabric Substrate

Figure 1. Assembly of a four layer textile composite showing the ideal gauge location

The mechanical properties of fabrics such as the bending rigidity, compressive modulus and bending modulus can be better characterised after locating the NA of the fabric $[15,16]$. To accurately estimate this NA position, any useful model must incorporate the tensile and in-plane compressive modulus of the fabric. Example models presented in [15-18] based on laminate theory and Kawabata evaluation system (KES) of fabrics showed that the NA position of woven or knitted fabrics is not close to the central axis and as a result fabrics cannot be treated as elastically homogenous. While these models are in agreement with the model presented in this work, they did not explore the influence of the elastic inhomogeneity of fabrics on the calculation of the NA positions of multi-layer laminated or printed fabrics.

This paper furthers these contributions by characterizing the elastic properties of woven fabrics by integrating the Pierce's bending theory of fabrics with the classical beam theory (CBT) to enable accurate modelling of the NA position and the bending characteristics of fabrics. The model is used to calculate the NA positions of four different textile composites, each consisting of a woven fabric substrate layer, a thermoplastic polyurethane (TPU) interface layer, the gauge layer and a TPU encapsulation layer as shown in figure 1. The TPU interface smooths the surface of the fabric by filling in the weave to improve the printing consistency of subsequent layers while the TPU encapsulation is a protective layer on top of the strain gauge. Controlling the thickness of the interface and encapsulation layers enables the position of the NA to be controlled and therefore it should be possible to ensure the printed active layer is located at the NA. The theoretically obtained NA positions from the model are experimentally validated by measuring the changes in the electrical resistance of piezoresistive strain gauges located within the printed composites during bending around a $5 \mathrm{~mm}$ radius. This model is shown to predict the NA axis of these textile composites with error margins of less than $1 \%$ in negative bending and $5 \%$ in positive bending. 


\section{Materials and properties}

\subsection{Fabric selection and characterisation}

Different textile blends of polyester, cotton, and lycra supplied by textile manufacturer Klopman International SRL were examined in this work. These fabrics (Bari, Polyester, Lagonda and Escalade) are currently standard textiles for garments and have different elastic properties which is useful when validating the model. The tensile modulus $E_{T}$ of the fabrics was obtained from a tensile test of rectangular specimens of each fabric in its weft (longitudinal yarn) and warp (orthogonal yarn) directions using a Tinius Olsen bench top tester. The width and gauge length of each test sample was $30 \mathrm{~mm}$ and $55 \mathrm{~mm}$. The measured tensile moduli are shown in Table 1 alongside other physical properties such as bending rigidity and fabric nonlinearity. The definitions and methods for obtaining these properties are discussed in Section 3.

Table 1. Physical Properties of fabrics

\begin{tabular}{|c|c|c|c|c|c|c|c|c|c|c|c|c|}
\hline Fabric & \multicolumn{2}{|c|}{ Bari } & \multicolumn{4}{|c|}{ Lagonda } & \multicolumn{4}{|c|}{ Escalade } & \multicolumn{2}{|c|}{${ }^{1}$ Polyester } \\
\hline Thickness $(\mu \mathrm{m})$ & \multicolumn{2}{|c|}{324} & \multicolumn{4}{|c|}{290} & \multicolumn{4}{|c|}{400} & \multicolumn{2}{|c|}{60} \\
\hline Twill & \multicolumn{2}{|c|}{$2 \times 1$} & \multicolumn{4}{|c|}{$2 \times 1$} & \multicolumn{4}{|c|}{$3 \times 1$} & \multicolumn{2}{|c|}{$2 \times 1$} \\
\hline Colour & \multicolumn{2}{|c|}{ Blue } & \multicolumn{4}{|c|}{ Cream } & \multicolumn{4}{|c|}{ Cream } & \multicolumn{2}{|c|}{ White } \\
\hline $\begin{array}{l}\text { Percentage Blend, \% } \\
\text { (Polyester/Cotton/Lycra) }\end{array}$ & \multicolumn{2}{|c|}{$65 / 35 / 0$} & \multicolumn{4}{|c|}{$30 / 40 / 30$} & \multicolumn{4}{|c|}{$16 / 46 / 48$} & \multicolumn{2}{|c|}{$100 / 0 / 0$} \\
\hline $\begin{array}{l}\text { Direction } \\
\text { Measurement }\end{array}$ & \multicolumn{2}{|c|}{ Warp / Weft } & \multicolumn{2}{|c|}{ Warp } & \multicolumn{2}{|c|}{ Weft } & \multicolumn{2}{|c|}{ Warp } & \multicolumn{2}{|c|}{ Weft } & \multicolumn{2}{|c|}{ Warp / Weft } \\
\hline${ }^{2}$ Bending orientation & Neg. & Pos. & Neg. & Pos. & Neg. & Pos. & Neg. & Pos. & Neg. & Pos. & Neg. & Pos. \\
\hline 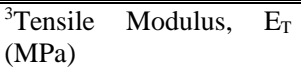 & \multicolumn{2}{|c|}{400} & \multicolumn{2}{|c|}{622} & \multicolumn{2}{|c|}{79} & \multicolumn{2}{|c|}{550} & \multicolumn{2}{|c|}{102} & \multicolumn{2}{|c|}{617} \\
\hline${ }^{4}$ Weight $\left(\mathrm{N} / \mathrm{m}^{2}\right)$ & \multicolumn{2}{|c|}{2.0} & \multicolumn{2}{|c|}{2.1} & \multicolumn{2}{|c|}{2.1} & & & & & & \\
\hline Overhang Length (m) & 0.041 & 0.043 & 0.055 & 0.066 & 0.037 & 0.045 & 0.059 & 0.077 & 0.05 & 0.059 & 0.033 & 0.035 \\
\hline $\begin{array}{l}{ }^{5} \text { Compressive Modulus, } \\
E_{C}(\mathrm{MPa})\end{array}$ & 2.0 & 2.0 & 6.84 & 12.95 & 2.37 & 4.82 & 4.4 & 11 & 3.26 & 5.92 & 55.53 & 70.34 \\
\hline $\begin{array}{l}\text { Bending Modulus, } B \\
(\mathrm{MPa})\end{array}$ & 6.4 & 7.6 & 22.82 & 39.2 & 6.87 & 12.4 & 15.4 & 34.1 & 9.28 & 15.3 & 131.4 & 157.34 \\
\hline $\begin{array}{l}{ }^{6} \text { Bending rigidity, } \quad G \\
(\mu \mathrm{Nm})\end{array}$ & 18.2 & 21.0 & 46.2 & 79.8 & 14.0 & 25.3 & 81.4 & 180.9 & 49.5 & 81.4 & 2.37 & 2.83 \\
\hline Fabric nonlinearity, $\eta$ & 0.016 & 0.019 & 0.037 & 0.067 & 0.087 & 0.157 & 0.028 & 0.062 & 0.091 & 0.150 & 0.213 & 0.255 \\
\hline $\begin{array}{l}\text { Fractional change in NA } \\
\text { position, } n\end{array}$ & 0.874 & 0.862 & 0.808 & 0.735 & 0.705 & 0.603 & 0.833 & 0.751 & 0.698 & 0.613 & 0.538 & 0.495 \\
\hline
\end{tabular}

${ }^{1}$ The cantilever test values were influenced by the surface properties of the fabric. The electrostatic force between the substrate and the inclined plane caused the fabric to bend which is not necessarily under its own weight.

${ }^{2}$ Bending orientation refers to the direction in which the fabric is bent i.e. positive or negative bending.

${ }^{3}$ The sample dimension for tensile test is $30 \mathrm{~mm}$ and $55 \mathrm{~mm}$.

${ }^{4}$ Weight per unit area of test sample of size $10 \mathrm{~cm} \times 1 \mathrm{~cm}$ is used for Pierce Cantilever test.

${ }^{5}$ Compressive Modulus is a measure of the fabric resistance/stiffness to elastic compression or contraction.

${ }^{6}$ Bending rigidity per unit width of the test sample.

\subsection{Screen printed ink selection and characterisation}

Screen printable UV-curable thermoplastic polyurethane (TPU) paste (Fabink-UV-IF-1004) and a thermally cured polymeric piezo-resistive carbon (PRC) paste (Fabink-TC-PolyPR1) were used in this study. The inks were supplied by Smart Fabric Inks Ltd. 
Fabink-UV-IF-1004 was used as the interface and encapsulation material due to its mechanical properties, such as its elastic modulus being comparable to the textile, and the strong adhesive bond to the textiles [12-15]. The PRC paste was used for the strain gauges because of its strain sensitivity (gauge factor of 5) and its low curing temperature of $100{ }^{\circ} \mathrm{C}$, which is compatible with the fabrics. The Tinuis Olsen benchtop tester was used to obtain the elastic modulus of the TPU layer. The elastic modulus of the PRC paste was measured using a Berkovich nano-indenter tip. Rectangular specimens of thickness $285 \mu \mathrm{m}$ and gauge length of $35 \mathrm{~mm}$ were used for the TPU tensile test based on the EN ISO 527-3 standard for testing plastic strips of less than $1 \mathrm{~mm}$ thickness. A $10 \mathrm{~mm}^{2}$ PRC specimen with an average thickness of $100 \mu \mathrm{m}$ was used for the nano-indenter measurements. The measured physical properties of these inks are given in Table 2.

Table 2. Physical Properties of inks

\begin{tabular}{lccc}
\hline \hline \multicolumn{1}{c}{ Pastes } & \multicolumn{2}{c}{ Fabink-TC-PolyPR1 $^{1}$} \\
& Fabink-UV-IF-1004 & Piezo-resistive carbon (PRC) \\
\hline \hline Supplier & 100 & Smart Fabinks Ltd \\
Elastic Modulus, $E_{T}(\mathrm{MPa})$ & 4.7 & 7050 \\
${\text { Viscosity at } 25^{\circ} \mathrm{C}(\mathrm{Pa} .)^{2}}^{2}$ & $18.9 \pm 0.5$ & 17.5 \\
Water Absorption (\%) & - & - \\
Gauge Factor (or gauge's sensitivity) & & 5 \\
\hline \hline
\end{tabular}

${ }^{1}$ The reduced modulus of the piezoresistive carbon is reported

${ }^{2}$ Shear rate $10 \mathrm{rpm}$

\section{Modelling}

Fabrics may in principle be characterised as beams of lengths, $l$, that satisfy the following conditions [19]:

$$
l>10 h \text { and } l>10 x
$$

Where $h$ and $x$ represent the thickness and width of the beam respectively. This ratio ensures flexibility, bending and drape characteristics which are typical of fabrics.

When in use, fabrics are subject to three principal forces: tensile/compressive, bending and torsional. Under bending forces, CBT states that a beam experiences maximum tensile and compressive stresses at its top and bottom surfaces respectively and a zero stress at the position of the neutral axis (NA) within the beam. The general predictive model equation for the position of NA, $\bar{y}$, for a single or multi-layered beam is given by [20]:

$$
\bar{y}=\frac{\sum_{i=1}^{n} E_{i} x_{i} h_{i}\left(2 \sum_{j=1}^{i} h_{j}-h_{i}\right)}{2 \sum_{i=1}^{n} E_{i} x_{i} h_{i}}
$$

Where $E_{i}, x_{i}$ and $h_{i}$ represent the elastic modulus, width and thickness of the " $i$ "th" layer of the beam respectively.

Paul et al. [14] showed that the direct application of (2) is inadequate for modeling the NA positions of multilayer textile composites because, unlike symmetrical beams, fabrics have unequal tensile and compressive moduli, $E_{T}$ and $E_{C}$ respectively. This difference in moduli makes the results from (2) very inaccurate and different to the empirically determined NA as demonstrated in [14]. The unequal tensile and compressive moduli also means the NA of a fabric does not coincide with its central axis [19].

Assuming the NA position is different to the central axis by distance $\delta$, the bending strain, $\varepsilon$, at an arbitrary distance $y$ from the central axis in pure bending (see figure 2) is given by [21]: 


$$
\varepsilon=\frac{\text { change in length at distance } y}{\text { undeformed length at distance } y}=\frac{(\rho+y-\delta) \theta-\rho \theta}{\rho \theta}=\frac{y-\delta}{\rho}
$$

Where $\rho$ and $\rho+(y-\delta)$ are the distances from the centre of curvature to the NA and to the arbitrary distance $y$ from the central axis. $\theta$ is the degree of curvature of the fabric and the un-deformed length of the fabric is the length of the fabric before bending.

Similarly, the bending stress in the fabric, $\sigma$, at this distance $y$ due to an applied force, $F$ on the cross sectional area, $A$ of the fabric is calculated from (4):

$$
\sigma=\frac{d F}{d A}=E \varepsilon=E \frac{y-\delta}{\rho}
$$

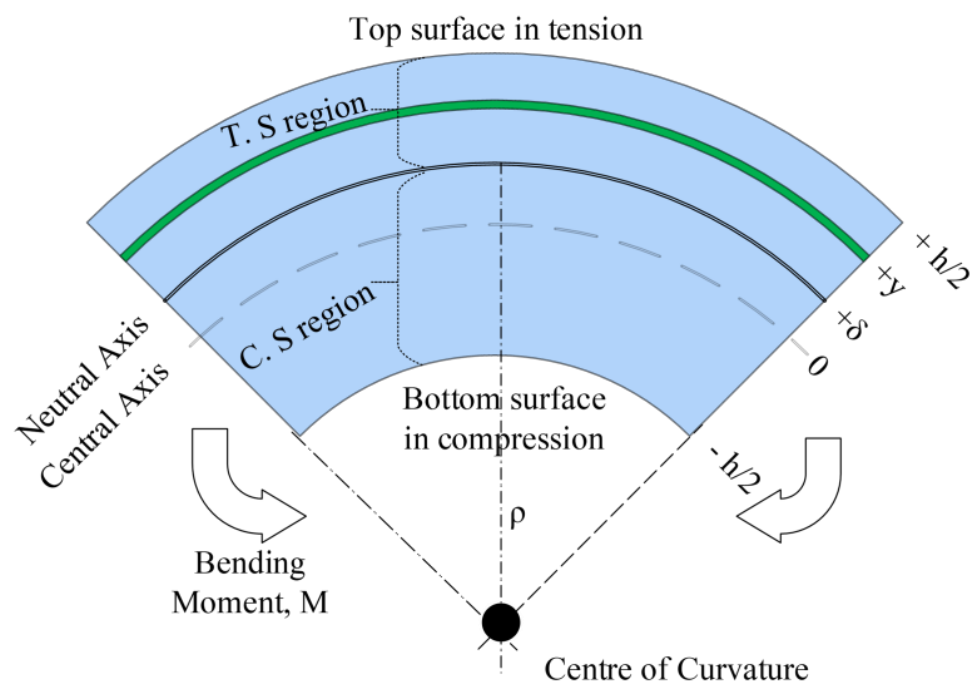

Figure 2. Deformation of a fabric under pure negative bending moments. TS and CS regions are the tensile and compressive stress regions respectively.

Where $E$ is the Young's modulus of the fabric which is either tensile $\left(E_{T}\right)$ or compressive $\left(E_{C}\right)$ when the fabric is bending.

At equilibrium, the net force, $F$ becomes zero:

$$
F=\int_{-\frac{h}{2}}^{\delta} E_{C}\left(\frac{y-\delta}{\rho}\right) x d A+\int_{\delta}^{\frac{h}{2}} E_{T}\left(\frac{y-\delta}{\rho}\right) x d A=0
$$

If the bi-modular ratio of the fabric, $\beta$ is defined as:

$$
\beta=\frac{E_{C}}{E_{T}}
$$

In fabrics, $\beta$ is always less than 1 because $E_{C}$ is invariably less than $E_{T}$. Inserting (6) into (5) enables the value of $\delta$ to be obtained from equation 7 :

$$
\delta=n\left(\frac{h}{2}\right) ; \quad n=\frac{1-\sqrt{\beta}}{1+\sqrt{\beta}}
$$

Where $n$ is the fractional change in the NA from its initial central axis position and $h$ is the thickness of the fabric. Equation (7) provides a more accurate analysis of the bending characteristics of a fabric when compared with equation (2). It also characterizes the effect of the difference in $E_{C}$ and $E_{T}$ on the NA position. 


\subsection{Effect of the difference in $E_{C}$ and $E_{T}$ on a fabric's bending characteristics}

If the normalized difference, $k$ between a fabric's tensile and compressive moduli, $E_{T}$ and $E_{C}$ is defined in (8):

$$
k=\frac{E_{T}-E_{C}}{E_{T}+E_{C}}=\frac{1-\beta}{1+\beta}
$$

Then the relationship between the fractional change in the NA position $n$ shown in (7) and $k$ is obtained by substituting (8) into (7) as:

$$
k=\frac{2 n}{n^{2}+1} \text { where } 0 \leq k \leq 1 ; 0 \leq n \leq 1
$$

A plot of (9) is shown in figure 3, which indicates that the distance of the NA increases nonlinearly from the central axis position with increasing $k$.

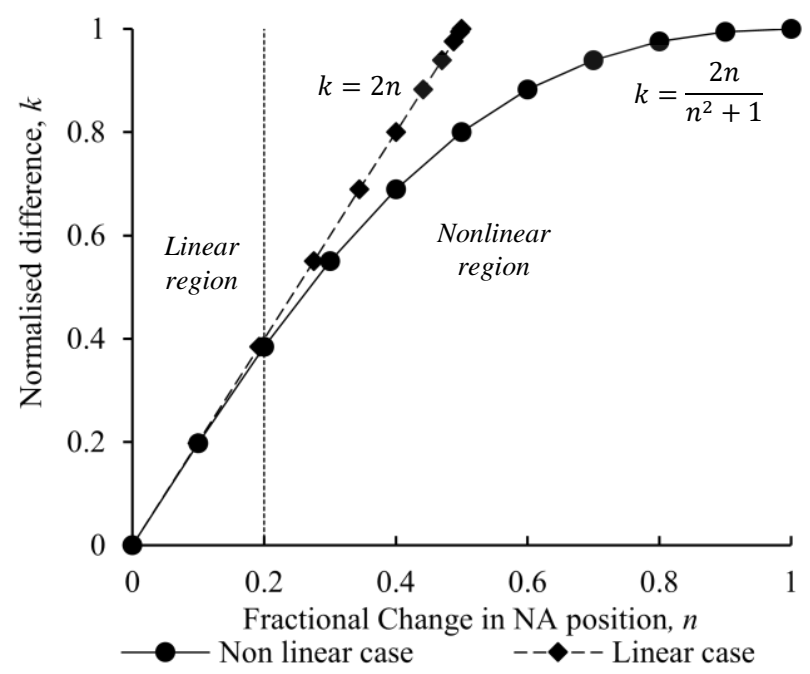

Figure 3. The effect of the normalised difference, $k$ in the compressive and tensile moduli on the fractional change in the NA, $n$ relative to the central axis of the fabric.

This nonlinearity changes the bending characteristics of the fabric by reducing its bending rigidity, $G$ which is a parameter used to describe the compliance or resistance of fabrics to bending. This is explained in the bending moment, $M$ equation of the fabric $[18,21]$ as follows:

$$
\begin{gathered}
M=\int_{-\frac{h}{2}}^{\delta} y E_{C}\left(\frac{y-\delta}{\rho}\right) x d A+\int_{\delta}^{\frac{h}{2}} y E_{T}\left(\frac{y-\delta}{\rho}\right) x d A=0 \\
M=\frac{\eta E_{T} I}{\rho} ; \quad \eta=(1-n)^{2} \\
G=\frac{\eta E_{T} I}{x} ; \quad I=\frac{x h^{3}}{12} \\
B=\eta E_{T}
\end{gathered}
$$

where $I$ is the moment of inertia of the fabric and $B$ is the bending modulus defined as the ability of the fabric to bend. $\eta$ is the nonlinearity introduced by the difference in the values of $E_{C}$ and $E_{T}$ which reduces the values of $B$ and $G$ in fabrics as shown in equations (11) and (12). This explains why woven fabrics 
are readily compliant to bending. The resistance of the fabric to bending is moderated by $\eta$; Pierce's cantilever bending test of fabrics, discussed below, was used to obtain the value of $\eta$ for the four different fabrics investigated in this work.

\subsection{Calculating fabric nonlinearity $(\eta)$}

Pierce's bending theory (described in figure 4) has been used to calculate the bending rigidity per unit width, $G$ of the fabric using equation 13 [23].

$$
G=W L^{3}\left(\frac{\cos (\alpha / 2)}{8 \tan \alpha}\right)
$$

where $W, L$ and $\alpha$ are the weight per unit area, overhang length and the bending angle of the fabric respectively.

The bending angle, $\alpha$ was set to $41.5^{\circ}$ to replicate the angle used in a standard commercial tool, the Shirley tester, which has been found to be optimum for this test but was unavailable to us for these tests [23]. Strips of dimensions $100 \mathrm{~mm} \times 10 \mathrm{~mm}$ of the four test fabrics were cut along their warp and weft directions. These were placed on a low-friction horizontal aluminium platform in the direction parallel to the longer dimension of the strip. The overhang length, $l$, used to calculate $G$ for positive and negative bending (shown in Table 1) was obtained by turning the fabric over and testing both sides. $l$ was measured at the point the fabric touches the inclined plane after bending.

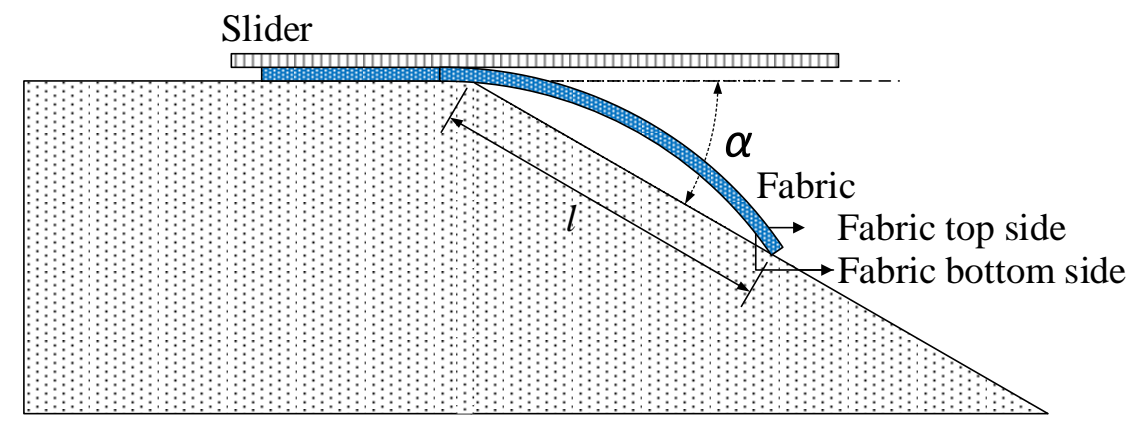

Figure 4. Pierce cantilever test of a $100 \mathrm{~mm} \times 10 \mathrm{~mm}$ specimen of fabric inclined at $41.5^{\circ}$ in negative bending. In positive bending, the top side of the fabric touches the incline plane.

Since $G$ varies under positive and negative bending, then from (11) it is clear $\eta$ also changes with bending direction. By equating (11) and (13), the values of $\eta$ corresponding to the $G$ values are calculated from:

$$
\eta=G \frac{x}{E_{T} I}
$$

The positive and negative bending values of $\eta$ were used to calculate $B$, and $E_{C}$ (reported in Table 1) using equations (6), (10) and (12). These values are more than $10^{2} \mathrm{MPa}$ lower than the $E_{T}$ values which reflects the compliance of the fabrics in bending.

\subsection{Effect of the fabric nonlinearity on the NA position}

The distance $\delta$ between the NA and the central axis of the four fabrics (reported in Table 1) was calculated from equations (7) and (10) using the nonlinearity values $\eta$ obtained in the cantilever test. Figure 5 shows the variation in $\delta$ plotted as normalised distance, $\delta_{n}$, where 0 equates to the central axis position and $100 \%$ is either surface of the fabric. The value of $\delta_{n}$ calculated from equation 15 increases 
as $\eta$ decreases as shown. Due to the significant difference between $E_{C}$ and $E_{T}$, the NA can in fact be located close to the surface of the fabric. Normalised distance has been converted into the actual position for each of the test fabrics in Table 3 .

$$
\delta_{n}(\%)=\frac{\text { Central axis }-N A}{N A} \times 100
$$

The graph also shows that, where a fabric has different tensile modulus $E_{T}$ along the warp and weft directions, the NA position is different for the warp and weft directions in both the positive and bending cases. Hence the Lagonda and Escalade fabrics have 4 data points in figure 5 which was plotted using Excel spreadsheet where all the values were computed. Where $E_{T}$ is the same along the warp and weft directions (i.e. the polyester and Bari fabrics), the NA position is identical in the warp and weft directions. The Lagonda and Escalade fabrics are comprised of high modulus polyester cotton and stretchable lycra yarns in the warp and weft weaves respectively and therefore have different tensile moduli in these directions.

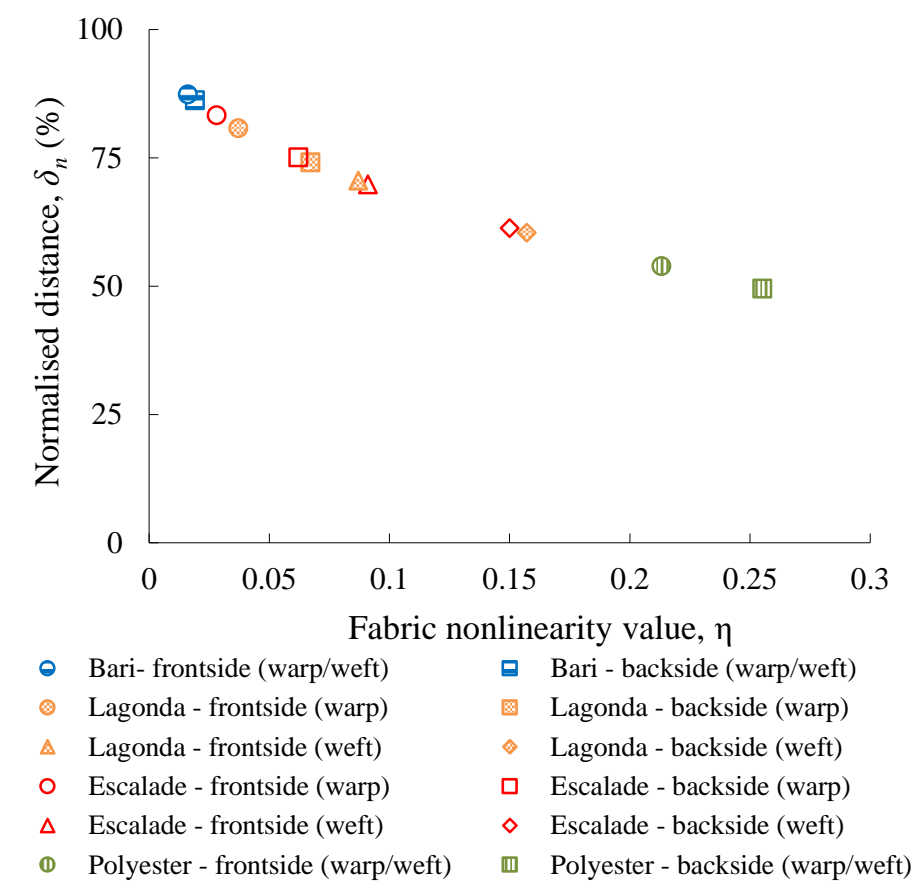

Figure 5. Relationship between the fabric nonlinearity, $\eta$ and the normalised NA distance, $\delta_{n}$ from the central axis of the fabrics. The top and bottom sides denote negative and positive bending of the fabrics respectively.

Figure 6 shows the influence of the direction of bending of the fabric where the top surface is defined as the location of the printed tracks. As shown in figure $6, \delta$ is always negative but its position changes depending on the direction of bending as is also shown in figure 5 .

\subsection{Effect of fabric nonlinearity in the modelling of the NA of screen printed e-textiles}

As soon as the printed layers are deposited onto the top surface of the fabric, the NA position will change from that shown in figure 6 . This new composite NA position is determined by equation 2 when the nonlinearity of the fabric is incorporated by considering the fabric as 

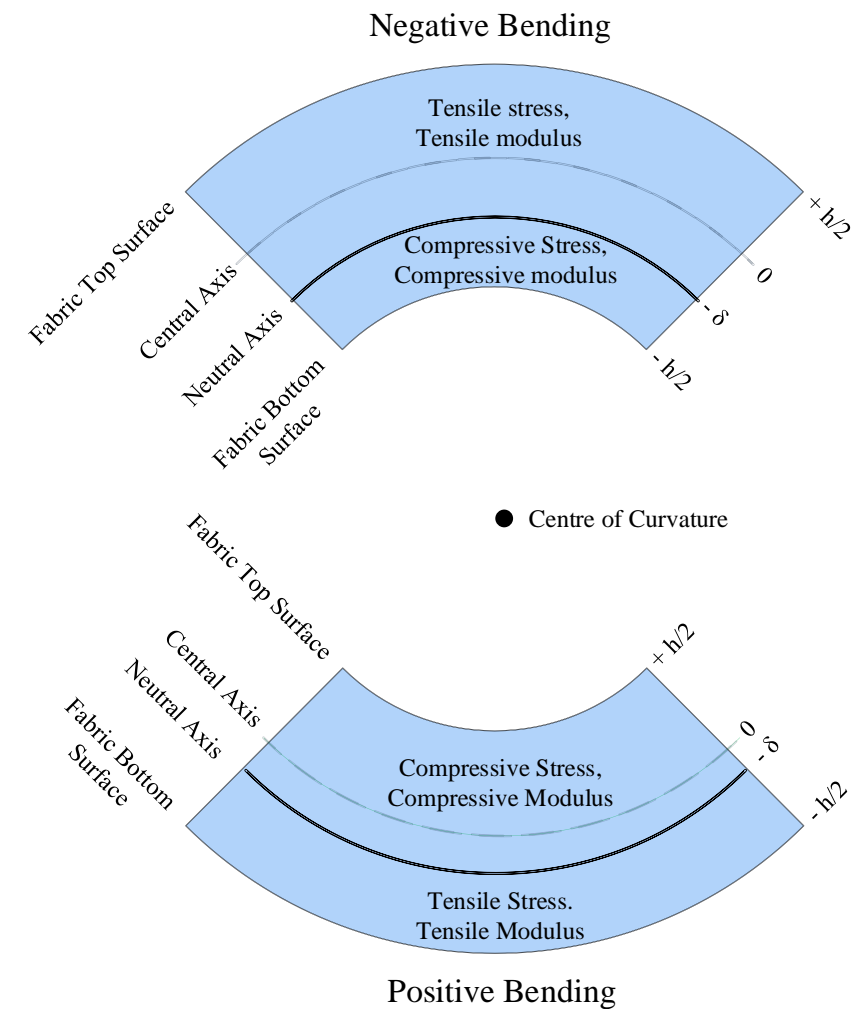

Figure 6. NA positions of a fabric in negative and positive bending.

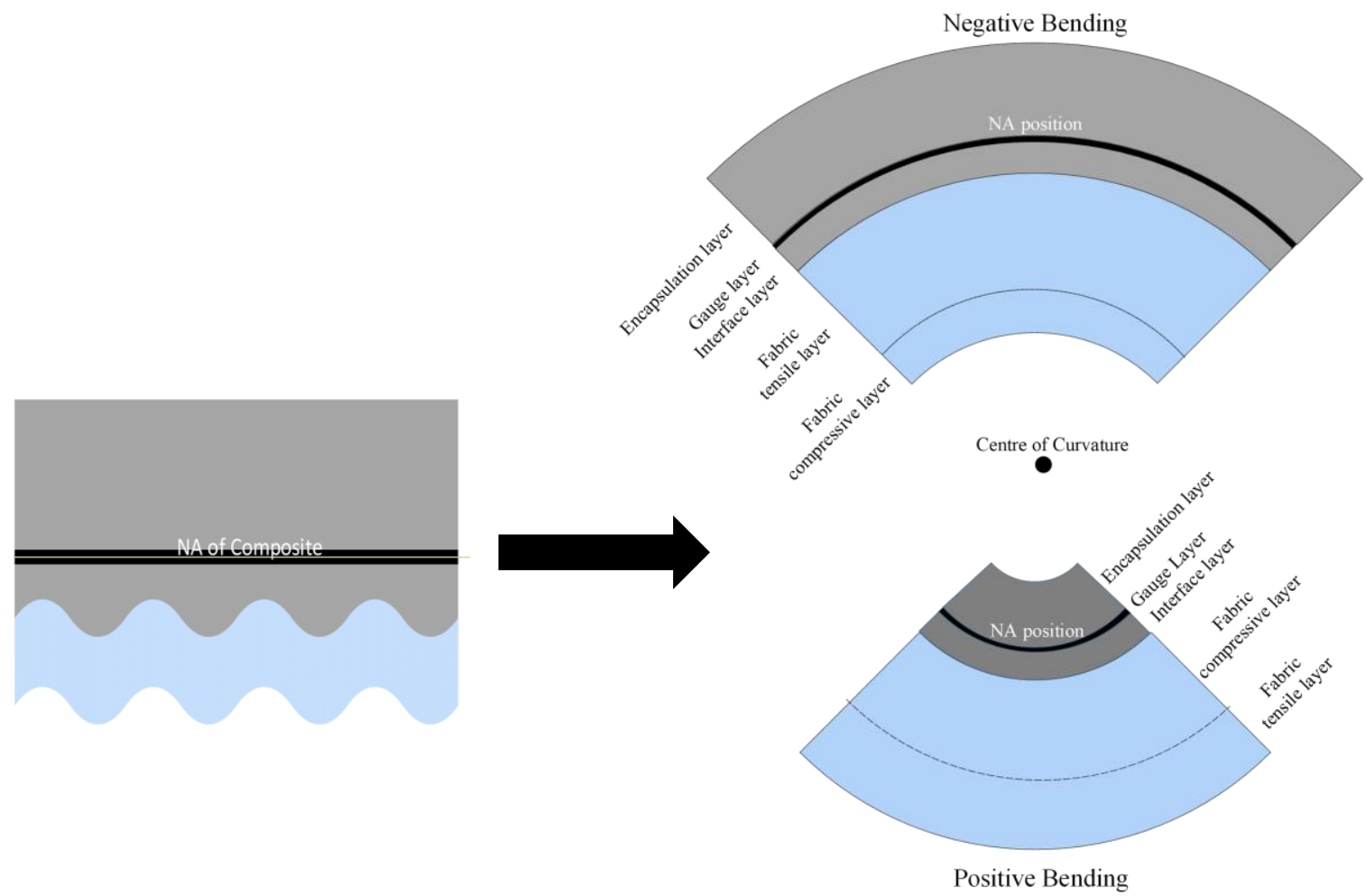

Figure 7. The five layer equivalent (right) of a four layer e-textile (left) in negative and positive bending orientations due to the nonlinearity of fabrics. NA position is at the gauge layer. 
separate layers with different moduli. This transforms the composite into a five layer structure (figure 7) where the fabric is split into its compressive and tensile regions which are characterized by the $E_{C}$ and $E_{T}$ values of the fabric respectively. These regions are bounded by the NA of the fabric which can be calculated from equation (16) where its thickness, $h$ and the fractional change in the NA position, $n$ of the fabric is shown in Table 1 . The bottom fabric layer has a thickness equivalent to the calculated fabric NAs shown in Table 3 for positive and negative bending orientations.

$$
\begin{aligned}
\text { Fabric } N A & =\text { Central axis }(C A)-\delta \\
& =(1-n) \frac{h}{2}
\end{aligned}
$$

\subsection{Optimizing printed film thicknesses to locate the NA of screen printed e-textiles}

In [12-14], the durability of screen printed e-textiles was improved by empirically controlling the film thicknesses of the interface and encapsulation layers so that the active layer is located on the NA. Since the interface thickness is usually initially obtained from experiment [10-14], the NA position is obtained by summing the fabric and interface thicknesses. Based on the model in section 3.4, the optimum thickness, $h_{T}$, of encapsulation that positions the gauge layer on this NA can therefore be calculated from equation (2). The $h_{T}$ values for the composites of the four different fabrics investigated in this work are shown in Table 4. The results show that different $h_{T}$ values are needed in negative and positive bending directions, and that the $h_{T}$ value in positive bending is smaller. This means the composites have different NA positions in these bending directions. It also clearly contradicts the results from the initial model without fabric nonlinearity which suggested the composites have the same NAs in all bending directions.

\begin{tabular}{|c|c|c|c|c|c|}
\hline \multirow{3}{*}{ Fabrics } & \multirow{3}{*}{$\begin{array}{c}\text { Central Axis } \\
\text { (Warp/Weft) } \\
\mu \mathrm{m}\end{array}$} & \multicolumn{4}{|c|}{$\mathrm{NA}(\mu \mathrm{m})$} \\
\hline & & \multicolumn{2}{|c|}{ Warp direction } & \multicolumn{2}{|c|}{ Weft direction } \\
\hline & & Negative bending & Positive bending & Negative bending & Positive bending \\
\hline Bari & 162 & 20.4 & 22.4 & 20.4 & 22.4 \\
\hline Polyester & 30 & 13.9 & 15.2 & 13.9 & 15.2 \\
\hline Lagonda & 145 & 27.8 & 38.4 & 42.8 & 57.6 \\
\hline Escalade & 200 & 33.4 & 49.8 & 60.4 & 77.4 \\
\hline
\end{tabular}

Table 3. NA positions of investigated fabrics

\begin{tabular}{|c|c|c|c|c|c|c|c|c|c|c|c|c|}
\hline \multirow{4}{*}{$\begin{array}{l}\text { Fabric } \\
\text { Fabric Weave } \\
\text { Bending Orientation } \\
\text { Position of strain gauge / Neutral } \\
\text { axis }(\mu \mathrm{m})\end{array}$} & \multirow{2}{*}{\multicolumn{2}{|c|}{$\begin{array}{c}\text { Bari } \\
\text { Warp/Weft }\end{array}$}} & \multirow{2}{*}{\multicolumn{2}{|c|}{$\begin{array}{c}\text { Polyester } \\
\text { Warp/Weft }\end{array}$}} & \multicolumn{4}{|c|}{ Lagonda } & \multicolumn{4}{|c|}{ Escalade } \\
\hline & & & & & \multicolumn{2}{|c|}{ Warp } & \multicolumn{2}{|c|}{ Weft } & \multicolumn{2}{|c|}{ Warp } & \multicolumn{2}{|c|}{ Weft } \\
\hline & Neg. & Pos. & Neg. & Pos. & Neg. & Pos. & Neg. & Pos. & Neg. & Pos. & Neg. & Pos. \\
\hline & \multicolumn{2}{|c|}{433} & \multicolumn{2}{|c|}{169} & \multicolumn{4}{|c|}{399} & \multicolumn{4}{|c|}{509} \\
\hline $\begin{array}{l}\text { Optimum encapsulation, } h_{T}(\mu \mathrm{m}) \\
\text { (fabric nonlinearity not modelled) }\end{array}$ & \multicolumn{2}{|c|}{840} & \multicolumn{2}{|c|}{335} & \multicolumn{2}{|c|}{960} & \multicolumn{2}{|c|}{353} & \multicolumn{2}{|c|}{1165} & \multicolumn{2}{|c|}{510} \\
\hline $\begin{array}{l}\text { Optimum encapsulation, } h_{T}(\mu \mathrm{m}) \\
\text { (fabric nonlinearity modelled) }\end{array}$ & 799 & 292 & 295 & 217 & 886 & 450 & 315 & 220 & 1090 & 542 & 450 & 305 \\
\hline $\begin{array}{l}\text { Estimated printed encapsulation } \\
\text { thickness }(\mu \mathrm{m})\end{array}$ & 600 & 400 & 400 & 300 & 1000 & 600 & 1000 & 300 & 1000 & 600 & 1000 & 400 \\
\hline
\end{tabular}

Table 4. Encapsulation thickness for the FLC structures on all fabrics

\section{Empirical validation of the NA of screen printed e-textiles}


The composite structure in figure 7 was fabricated on Bari, Polyester, Lagonda and Escalade fabrics using the same screen printing process described in our previous work [12]. The printed strain gauges were aligned either along the warp or weft direction of the fabrics to confirm the effect of the elastic properties of the fabrics on the position of the NA.

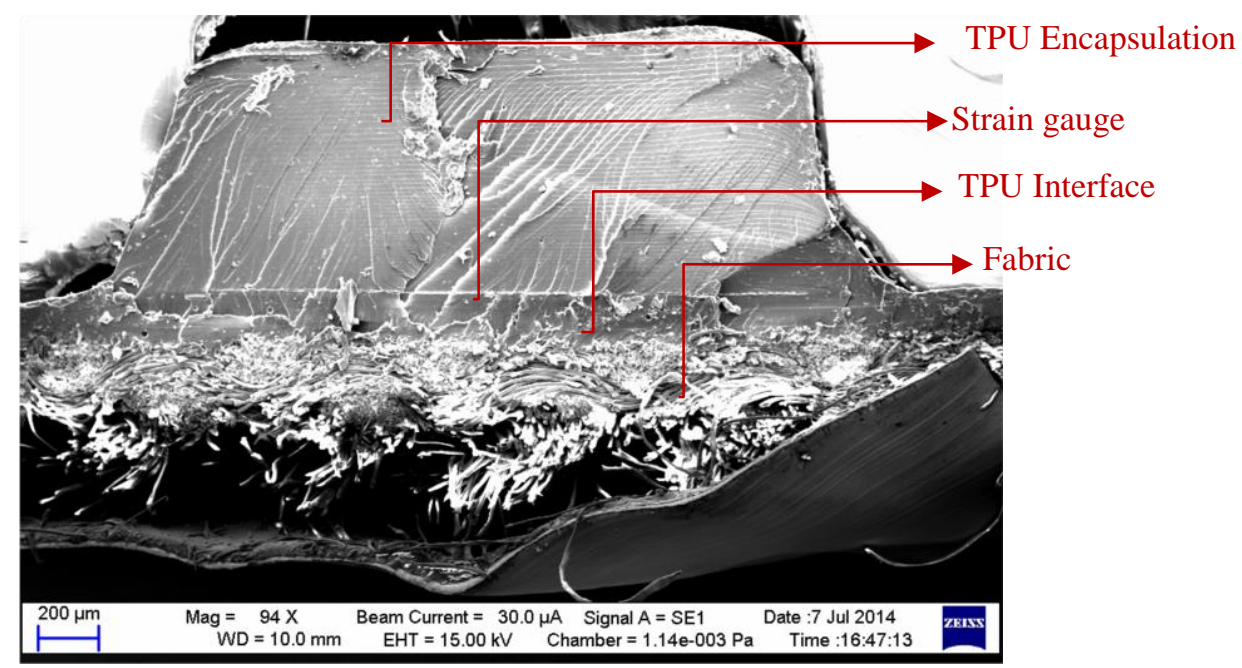

Figure 8. An SEM cross section of a strain gauge sandwiched between two TPU layers on Bari fabric

A cross section of the screen printed four layer e-textile composite is shown in figure 8 . The average thickness of the printed polyurethane interface layer on the four fabric types is $109 \mu \mathrm{m}$ while the encapsulation layer thickness was varied between $100 \mu \mathrm{m}$ and $800 \mu \mathrm{m}$.

\subsection{Bending test and results}

The calculated optimum thickness $h_{T}$ of encapsulation, shown in Table 4, was empirically verified from the resistance change of the printed gauges bent around a $5 \mathrm{~mm}$ radius rod as shown in figure 9 . The resistance change, $\Delta R$ in the gauges during bending (i.e. in-bending changes) was calculated from the resistance of the gauge before and during bending measured from a multimeter. This was averaged over two samples as reported in figure 10. In compression, $\Delta R<0$ and in tension $\Delta R>0$. The level of stress experienced by the gauge depends upon its promixity to the NA. When in close proximity, the stress is minimised and the change in resistance approaches zero i.e. $\Delta R \approx 0$.

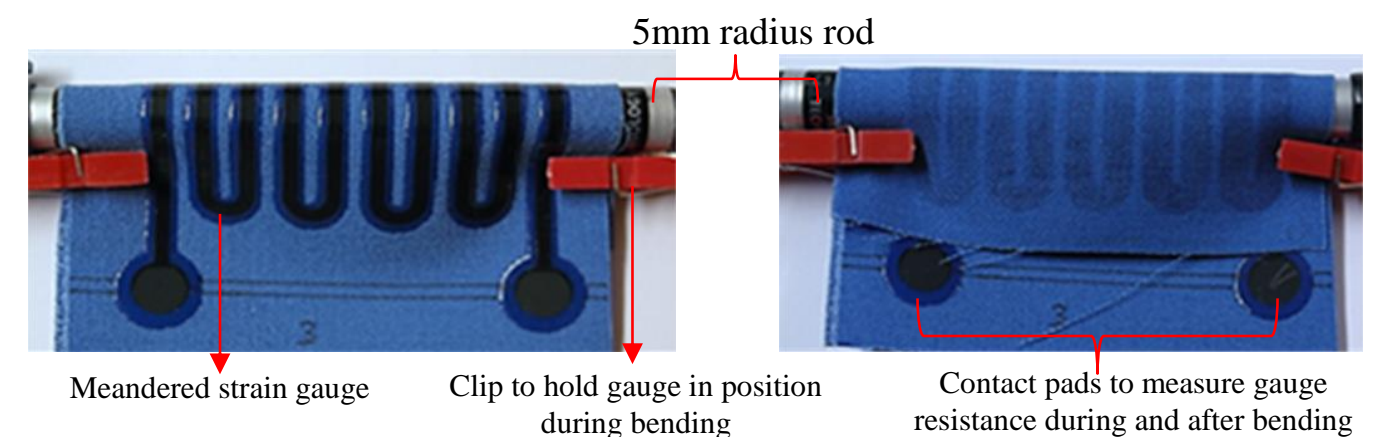

Figure 9. Testing of the strain gauge in Bari fabric in negative (left) and positive bending orientations (right). 

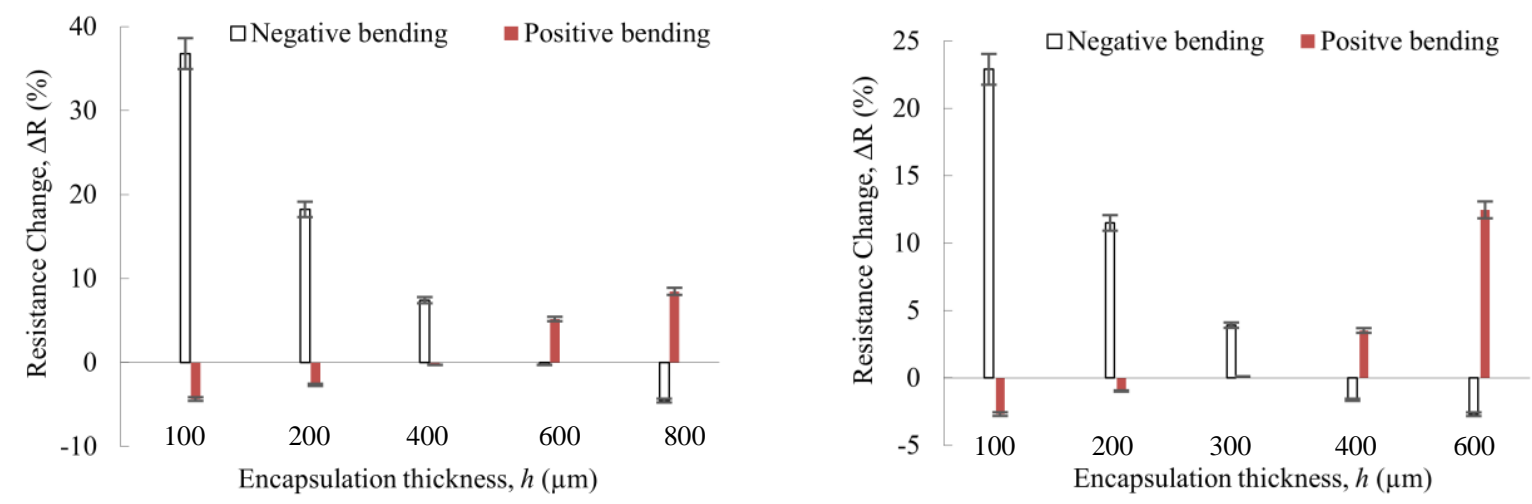

(a) Bari (left) and Polyester (right) composites for both weft and weave directions.
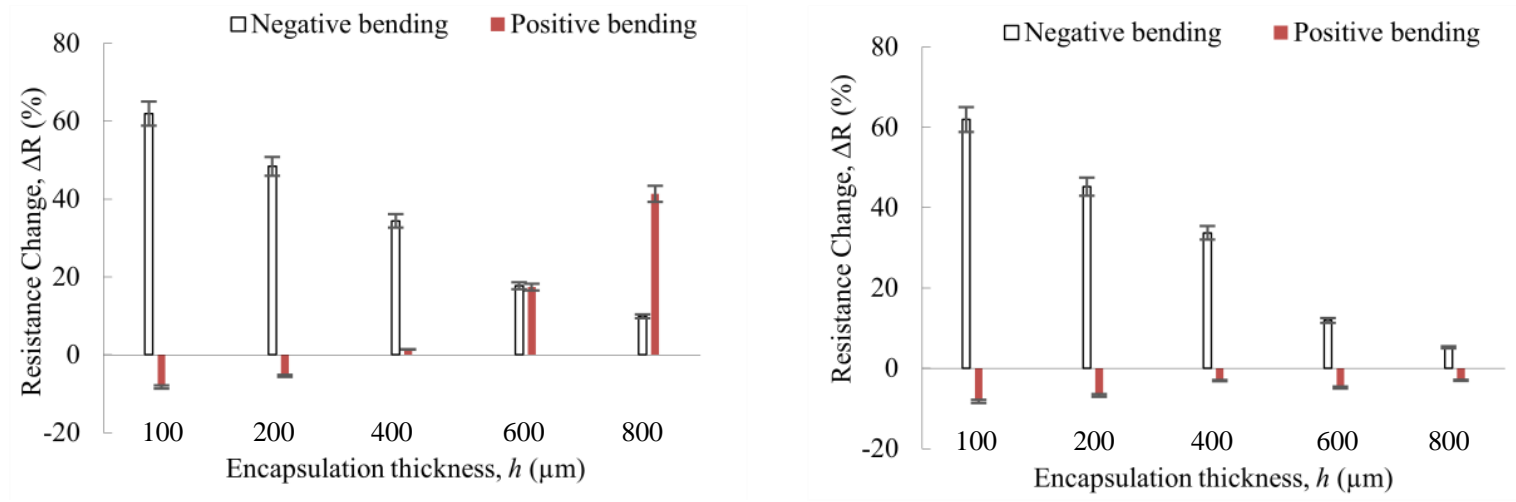

(b) Lagonda composites along the elastic weft (left) and inelastic warp (right) weave respectively.
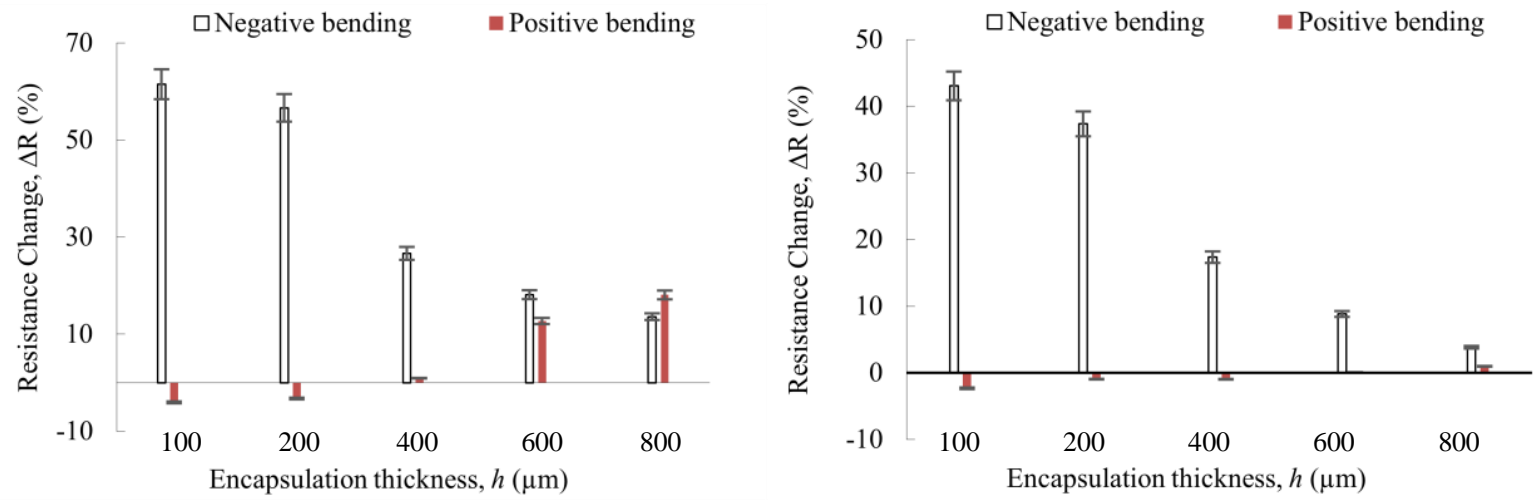

(c) Escalade composites along the elastic weft (left) and inelastic warp (right) weave respectively.

Figure 10. In-bending responses of strain gauges printed in FLC structures of the Bari, Polyester, Lagonda and Escalade e-textile composites due to positive and negative bending radius of $5 \mathrm{~mm}$. Optimum thickness, $h_{T}$ is achieved at the NA when $\Delta R \approx 0$.

A comparison of the theoretical $h_{T}$ values in Table 4 and the empirical $h_{T}$ values is presented in figure 10 , which shows that the theoretical $h_{T}$ value is closer to the empirical $h_{T}$ when the fabric nonlinearity was included. For example, the empirical $h_{T}$ for the Bari composite in figure 10a is approximately 400 $\mu \mathrm{m}$ in positive bending, and $600 \mu \mathrm{m}$ in negative bending. The theoretical $h_{T}=840 \mu \mathrm{m}$ in both bending directions predicted from the model without the fabric nonlinearity. This represents a percentage error of $110 \%$ in the optimum thickness for positive bending and $40 \%$ for negative bending. In the improved model (with fabric nonlinearity), these errors were reduced to $27 \%$ and $33 \%$ respectively. The improved 
model also agrees with the experimental results in that a different $h_{T}$ is required for positive and negative bending, and that the $h_{T}$ in positive bending is smaller. This trend is consistent for the gauges on all the fabrics.

Figures $10 \mathrm{~b}$ and $10 \mathrm{c}$ did however show unexpected behaviour for the gauges in negative bending on Lagonda and Escalade fabrics characterized by different elastic moduli in the weft and warp directions. The resistance changes in the gauges aligned along the weft direction (direction of low stiffness) of the fabrics closely mirrored the changes observed when the gauges were aligned along the warp direction of higher stiffness. This is thought to be because of the limiting effect of the interface layer on the bending dynamics of both fabrics. In woven fabrics, the warp and weft yarns are typically interlocked in a way that both yarns are still able to slide across each other, shear, stretch or contract about their woven position. The interface layer impregnates the fabric during printing and in some cases rigidly bonds the yarns together thereby preventing these movements. Consequently, the bending behaviour of the fabric when its yarns are interlocked by the interface is dominated by the elastic modulus of the stiffer yarn. This could also explain why the theoretical $h_{T}$ values predicted from the model are still not that close to the empirical equivalents. Hence the parameters used in the model were investigated further until it accurately predicted the optimum encapsulation thickness and this is discussed in section 4.2.

As predicted by the model described in section 3.3, the experimental result in figure 10a also showed two NA positions (i.e. where $\Delta R \approx 0$ ) in Bari and Polyester fabrics, each in positive and negative bending. Similarly in the Lagonda and Escalade fabrics, four NA.s were also observed as shown in Figure 10b and 10c respectively. The results confirm that these NA positions change with the bending direction and the elastic characteristics of the fabrics. Therefore to achieve durable screen printed e-textiles with such fabrics, printed electronics must be properly aligned along the fabric weave (warp or weft) direction in bending.

\subsection{Effect of the interface layer on the elastic characteristics of the screen printed composite}

To include the effect of the interface layer impregnating the fabric and binding the yarns, the properties of the fabric layer in the model must be modified. The tensile modulus of the interface impregnated fabric can be determined by tensile testing the fabric with a single interface layer (shown in Table 5). The optimum encapsulation thicknesses can then be recalculated from equation (2). The compressive modulus shown in table 5 was obtained empirically by printing encapsulation thicknesses ranging from $100 \mu \mathrm{m}$ to $800 \mu \mathrm{m}$, and the value of $E_{C}$ was chosen by making sure the calculated theoretical distances of the gauges from the NA using equation 15 correlated with the degree of change in electrical resistance of the strain gauges. The new tensile and compressive moduli were used to calculate the new optimum encapsulation thicknesses shown in Table 6.

The new theoretical $h_{T}$ value obtained by modelling the interface effect is closer to the estimated printed encapsulation thickness compared with the previous model as shown in Table 6. The percentage error in the encapsulation thickness for example in Bari fabric is reduced to $0.8 \%$ and $4.5 \%$ in negative and positive bending respectively. This is an improvement on the $33 \%$ and $27 \%$ error obtained with the previous model. This trend is consistent with results from the Lagonda and Escalade composites except during negative bending along the weft direction of the fabrics. As explained in section 4.1, the composites behave as though they were bent along the stiffer warp direction of the fabrics hence, the tensile and compressive moduli of the fabrics along the weft were replaced with the warp direction equivalents. The new $h_{T}$ values for Lagonda and Escalade during negative bending along the weft direction are $851 \mu \mathrm{m}$ and $980 \mu \mathrm{m}$ respectively. These values are above the thickest printed encapsulation at $h_{T}=800 \mu \mathrm{m}$ and agree with the experimental results which show that the printed gauges are not yet positioned on the NA with $h_{T}=800 \mu \mathrm{m}$ but that the general trend in the graph shows it is approaching the NA with increased encapsulation. 
Table 5. Elastic modulus of UV-IF-1004 impregnated fabrics

\begin{tabular}{|c|c|c|c|c|c|c|c|}
\hline \multirow{2}{*}{\multicolumn{2}{|c|}{ Fabrics }} & \multirow{2}{*}{$\begin{array}{c}\text { Fabric + UV-IF- } \\
1004 \text { average } \\
\text { thickness }\end{array}$} & \multirow[t]{2}{*}{ "Width } & \multirow[t]{2}{*}{$\begin{array}{l}\text { Gauge } \\
\text { length }\end{array}$} & \multirow{2}{*}{$\begin{array}{c}{ }^{1} \text { Average } \\
\text { tensile modulus, } E_{T} \text { of } \\
\text { impregnated fabric }\end{array}$} & \multicolumn{2}{|c|}{$\begin{array}{c}{ }^{2} \text { Approximate compressive modulus, } \\
E_{C} \text { of impregnated fabric in }\end{array}$} \\
\hline & & & & & & $\begin{array}{l}\text { Negative } \\
\text { bending }\end{array}$ & $\begin{array}{l}\text { Positive } \\
\text { bending }\end{array}$ \\
\hline \multicolumn{2}{|c|}{ Bari } & $348 \mu \mathrm{m}$ & $1 \mathrm{~cm}$ & $3.5 \mathrm{~cm}$ & $220 \pm 19 \mathrm{MPa}$ & \multicolumn{2}{|c|}{$80 \mathrm{MPa}$} \\
\hline \multirow[t]{2}{*}{ Escalade } & Warp & $441 \mu \mathrm{m}$ & & & $443 \pm 14 \mathrm{MPa}$ & $90 \mathrm{MPa}$ & $60 \mathrm{MPa}$ \\
\hline & Weft & $434 \mu \mathrm{m}$ & $0.4 \mathrm{~cm}$ & $3 \mathrm{~cm}$ & $56 \pm 3 \mathrm{MPa}$ & $90 \mathrm{MPa}$ & $52 \mathrm{MPa}$ \\
\hline \multicolumn{2}{|c|}{ Polyester } & $75 \mu \mathrm{m}$ & & & $615 \pm 44 \mathrm{MPa}$ & \multicolumn{2}{|c|}{$95 \mathrm{MPa}$} \\
\hline
\end{tabular}

${ }^{1}$ Fabric-interface modulus was averaged over four samples.

${ }^{2}$ Values were deduced from the electrical resistance changes of the strain gauges

Table 6. Optimum thicknesses of encapsulation for the FLC structures due to the interface effect

\begin{tabular}{|c|c|c|c|c|c|c|c|c|c|c|c|c|}
\hline \multirow{4}{*}{$\begin{array}{l}\text { Fabric } \\
\text { Fabric Weave } \\
\text { Bending Orientation } \\
\text { Old encapsulation thickness, } \\
h_{T}(\mu \mathrm{m})\end{array}$} & \multirow{2}{*}{\multicolumn{2}{|c|}{$\begin{array}{c}\text { Bari } \\
\text { Warp/Weft }\end{array}$}} & \multirow{2}{*}{\multicolumn{2}{|c|}{$\begin{array}{c}\text { Polyester } \\
\text { Warp/Weft }\end{array}$}} & \multicolumn{4}{|c|}{ Lagonda } & \multicolumn{4}{|c|}{ Escalade } \\
\hline & & & & & \multicolumn{2}{|c|}{ Warp } & \multicolumn{2}{|c|}{ Weft } & \multicolumn{2}{|c|}{ Warp } & \multicolumn{2}{|c|}{ Weft } \\
\hline & Neg. & Pos. & Neg. & Pos. & Neg. & Pos. & Neg. & Pos. & Neg. & Pos. & Neg. & Pos. \\
\hline & 799 & 292 & 295 & 217 & 886 & 450 & 315 & 220 & 1090 & 542 & 450 & 305 \\
\hline $\begin{array}{l}\text { New encapsulation thickness } \\
\text { value, } h_{T}(\mu \mathrm{m})\end{array}$ & 605 & 418 & 295 & 217 & 852 & 525 & 280 & 245 & 980 & 580 & 406 & 373 \\
\hline $\begin{array}{l}\text { Estimated printed } \\
\text { encapsulation thickness }(\mu \mathrm{m})\end{array}$ & 600 & 400 & 400 & 300 & 1000 & 600 & 1000 & 300 & 1000 & 600 & 1000 & 400 \\
\hline
\end{tabular}

The bending behaviour of the polyester fabric composites was inaccurately characterised by the model. Empirical results indicate that the elastic property of the polyester fabric when incorporated in the NA position tests is different from the one obtained from the tensile test. The calculated tensile and compressive modulus values of the fabric based on the measured resistance changes in the gauges printed on it are $2.25 \mathrm{GPa}$ and $95 \mathrm{MPa}$ respectively. These values, in particular the tensile modulus, clearly contradict the measured value of $617 \mathrm{MPa}$ from the Tinius Olsen tensile tester used to characterize the polyester fabric in the model. This anomaly underlines the unusual bending characteristics of fabrics and the need for methods that enable the correct characterization and measurements of the mechanical properties of fabrics.

\section{Conclusions}

This paper focused on developing and empirically validating a theoretical model for the neutral axis (NA) position of a screen printed e-textile by characterizing its elastic behavior in bending. The modeling and experimental evaluation of screen printed composites of four fabrics, Bari, Polyester, Lagonda, and Escalade of different elastic properties yielded the following conclusions.

The positioning of printed conductive tracks or electronic circuits in proximity to the neutral axis of a screen printed e-textile can minimize the level of stress experienced. As a consequence, the durability of the e-textile is enhanced. By controlling the thickness of the printed interface and encapsulation layers using the printing process, the neutral axis can coincide with the position of the printed electronics in the screen printed e-textile. The interface layer thickness for a fabric is a function of the printing parameters and screen properties and hence is at a predetermined value. So the optimum thickness, $h_{T}$ of the encapsulation required to ensure the position of the printed electronics coincides with the NA can be calculated from modelling the bending behavior of the e-textile. However, the bending behavior of the screen printed e-textile is complicated by the unusual mechanical properties of the fabric substrate. The different elastic moduli of fabrics in tension and compression affects the position of the NA in the 
fabric and this ultimately affects the NA position of the e-textile whenever the bending direction or the elastic modulus of the fabric changes. Therefore any useful theoretical model needs to characterize the compressive and tensile elastic properties of the fabric to find the NA position of the e-textile.

Furthermore, during screen printing, the printed interface layer impregnates the fabric and restricts the movement of the woven yarns after curing. This alters the elastic property and makes the fabric to behave like a bimetallic strip wherein the stiffer yarn in the fabric controls the bending behavior. This consequently affects the NA position of a screen printed e-textile due to the change in the elastic behaviour of the fabric. The model must characterize this effect to properly locate the NA to the etextile. While this paper demonstrated the modelling and empirical validation of the NA position, the results from the model are not perfect in the case of the polyester fabric. The properties of the polyester fabric are difficult to determine experimentally and therefore the values used in the model are not as accurate as for the other fabrics.

Both modeling and experimental results however indicate that screen printed e-textiles fabricated with fabrics that are characterized with the same elastic modulus along the warp and weft directions such as Bari and Polyester fabrics, will have two NA positions, one each in positive and negative bending. This means that the position of the integrated electronics must be a compromise between the requirements of the two bending directions. For the Bari and polyester fabrics, the compromise encapsulation thickness should be $h_{T}=500 \mu \mathrm{m}$ and $h_{T}=350 \mu \mathrm{m}$ respectively. The use of fabrics with different elastic modulus along the warp and weft weave directions such as Lagonda and Escalade fabrics, will result in four different NA positions within the e-textile, each with a different pair of NAs in positive and negative bending along the warp and weft directions of the fabrics. The durability of electronics integrated on such fabrics are best when the application of the printed e-textile is limited to bending along either the warp or weft direction so that the electronics is positioned on NA in any bending orientation or close to the NAs of both bending orientations. Hence the durability of such fabrics can be improved by comprising on the position of the integrated electronics such that its distance from the four NAs is reduced to a minimum. In applications where the fabric is bent or stretched in a particular direction during manufacturing, such as architectural or furnishing fabrics, the orientation of the prints can be determined by the area of the fabric which will experience the highest strain. In general, the optimal thickness, $h_{T}$ of the TPU interface and encapsulation layers required to sandwich screen printed devices on the NA is primarily controlled by the elastic modulus of these materials. Depending on the choice of fabric, $h_{T}$ can be as high as $1 \mathrm{~mm}$ when using Fabink-UV-IF-1004 $\left(E_{T}=100 \mathrm{MPa}\right)$ as the TPU interface and encapsulation. While the $h_{T}$ can be minimised by reducing fabric thickness or by increasing the $E_{T}$ of the TPU layers, it is recommended that the printed film is not allowed to dominate the e-textile. This would minimise its effect on the feel and comfort of the fabric, and the awareness of the wearer to the printed films [12].

This model is useful for improving the durability of printed layers on fabrics despite the challenges of working with textile substrates. To prevent or minimise the risk of delamination as the TPU thickness increases in e-textile devices, it would be useful in the future to determine the shear stress between the fabric and the printed films. When combined with suitable experimental data, this could provide an indication of the adhesive or bond strength and the point of at which the interface layer will delaminate from the fabric.

\section{Acknowledgements}

The authors thank the EPSRC for supporting this research with grant reference EP/I005323/1. The data for this paper can be found at DOI: 10.5258/SOTON/D0366. 


\section{References}

[1] Cho G, Lee S and Cho J 2009 Review and reappraisal of smart clothing International Journal of Human-Computer Interaction 25 582-617

[2] Sekitani T, Nakajima H, Maeda H, Fukushima T, Aida T, Hata K and Someya T 2009 Stretchable active-matrix organic light-emitting diode display using printable elastic conductors Nature materials 8494

[3] De Vos M, Torah R, Glanc-Gostkiewicz M and Tudor J 2016 A complex multilayer screen-printed electroluminescent watch display on fabric Journal of Display Technology 12 1757-1763

[4] Kim Y, Kim H and Yoo H J 2010 Electrical characterization of screen-printed circuits on the fabric IEEE transactions on advanced packaging 33 196-205

[5] Paul G, Torah R, Beeby S and Tudor J 2014 The development of screen printed conductive networks on textiles for biopotential monitoring applications Sensors and Actuators A: Physical 206 35-41

[6] Merritt C R, Nagle H T and Grant E 2009 Fabric-based active electrode design and fabrication for health monitoring clothing IEEE Transactions on information technology in biomedicine $\mathbf{1 3}$ 274-280

[7] Karaguzel B, Merritt C R, Kang T, Wilson J M, Nagle H T, Grant E and Pourdeyhimi B 2008 Utility of nonwovens in the production of integrated electrical circuits via printing conductive inks Journal of the Textile Institute 99 37-45

[8] Kazani I, Hertleer C, De Mey G, Schwarz A, Guxho G and Van Langenhove L 2012 Electrical conductive textiles obtained by screen printing Fibres \& Textiles in Eastern Europe 20 57-63

[9] Merilampi S, Laine-Ma T and Ruuskanen P 2009 The characterization of electrically conductive silver ink patterns on flexible substrates Microelectronics reliability 49 782-790

[10] Yang K, Torah R, Beeby S and Tudor J 2013 Flexible and washable conductive textile achieved by screen printing for smart fabric applications LOPE-C - Large-Area, Organic and Printed Electronics Convention (Munich)

[11] Yang K, Torah R, Wei Y, Beeby S and Tudor J, 2013 Waterproof and durable screen printed silver conductive tracks on textiles Textile Research Journal 83 2023-2031.

[12] Komolafe A O, Torah R N, Yang K, Tudor J and Beeby S P 2015 Durability of screen printed electrical interconnections on woven textiles In Electronic Components and Technology Conference (ECTC) 2015 IEEE 65th 1142-1147

[13] Komolafe A, Torah R, Tudor J and Beeby S 2017 Improving the durability of screen printed conductors on woven fabrics for e-textile applications In Multidisciplinary Digital Publishing Institute Proceedings 1613

[14] Paul G, Torah R, Yang K, Beeby S and Tudor J 2014 An investigation into the durability of screenprinted conductive tracks on textiles Measurement Science and Technology 25025006

[15] Kim K, Inui S and Takatera M 2013 Prediction of bending rigidity for laminated weft knitted fabric with adhesive interlining Textile Research Journal 83 937-946

[16] Kim K, Inui S and Takatera M 2013 Bending rigidity of laminated fabric taking into account the neutral axes of components Textile Research Journal 83 160-170

[17] Nakura M and Mizutani M 1988 On the position of the neutral plane in the woven fabrics to which the pure bending is applied Journal Japan Res Assoc Textil End Uses 29 193-198

[18] Yu W R, Zampaloni M, Pourboghrat F, Chung K and Kang T J 2005 Analysis of flexible bending behavior of woven preform using non-orthogonal constitutive equation Composites Part A: Applied Science and Manufacturing 36 839-850.

[19] Huston R and Josephs H 2009 Practical stress analysis in engineering design CRC Press New York USA $103-110$

[20] Ballas R G 2007 Theory of the Static Behavior of Piezoelectric Beam Bending Actuators. Piezoelectric Multilayer Beam Bending Actuators: Static and Dynamic Behavior and Aspects of Sensor Integration Springer-Verlag Berlin Heidelberg 47-76

[21] Hearn E J 1997 Mechanics of Materials Published by Butterworth-Heinemann, London 62-88 
[22] Kenkare N and May-Plumlee T 2005 Evaluation of drape characteristics in fabrics International Journal of Clothing Science and Technology 17 109-123

[23] Sun M N 2008 A new tester and method for measuring fabric stiffness and drape Textile Research Journal 78 761-770 